\title{
5 A Brief Review of Microwave Heating
}

\subsection{Introduction}

Microwave frequencies occupy the portion of the electromagnetic spectrum $(300 \mathrm{MHz}$ to $300 \mathrm{GHz})$ that lies between VHF radio-waves and thermal infrared. Their application falls into two categories, depending on whether the wave is used to transmit information or energy. The first category includes terrestrial and satellite communication links, radar, radio-astronomy, microwave thermography, material permittivity measurements, and so on (Adamski \& Kitlinski, 2001). The second category of applications is associated with microwave heating and wireless power transmission. In the case of microwave heating, there is usually no signal modulation and the electromagnetic wave interacts directly with solid or liquid materials.

"It has long been known that an insulating material can be heated by applying energy to it in the form of high frequency electromagnetic waves" (Metaxas \& Meredith, 1983, pp. 5). Industrial microwave heating has been used since the 1940's (Metaxas \& Meredith, 1983, pp.5). The initial experiments with microwave heating were conducted by Dr. Percy Spencer in 1946, following a serendipitous accident while he was testing a magnetron (Gallawa, 1998). Although Spencer was not the first to observe that microwave energy could impart heat to materials, he was the first to systematically study it. Since then many heating, drying, thawing (Liu, Marchant, Turner, \& Vegh, 2003) and medical applications (Bond, Li, Hagness, \& Van Veen, 2003) have been developed.

One key benefit of microwave heating, over conventional convective heating, is speed. The origin of this speed is the volumetric interactions between the microwave's electric field and the material. In contrast, convective heat transfer propagates from the surface into the material, with the final temperature profile depending on the material's thermal diffusion properties (Holman, 1997) and the influence of moisture transport, which often hinders the convective heating process (Crank, 1979).

The factors that contribute to microwave heating include: the physical and chemical structure of the heated material; the frequency of the microwaves (Van Remmen, Ponne, Nijhuis, Bartels, \& Herkhof, 1996); in some cases, such as wood, the orientation of the electrical field relative to the structure of the dielectric material (Torgovnikov, 1993, pp. 13-17); reflections from the inter-facial surface of the heated material (Adamski \& Kitlinski, 2001); electric field strength (Van Remmen et al., 1996); the geometry of the microwave applicator (Metaxas \& Meredith, 1983); the geometry, size, electrical and thermal properties of the dielectric material (Brodie, 2008; Perre \& Turner, 1999; Zhao, Turner, \& Torgovnikov, 1998); the exposure time; and the moisture content of the dielectric material (Crank, 1979; Torgovnikov, 1993). This chapter briefly explores electromagnetic heating with a focus on some of the 


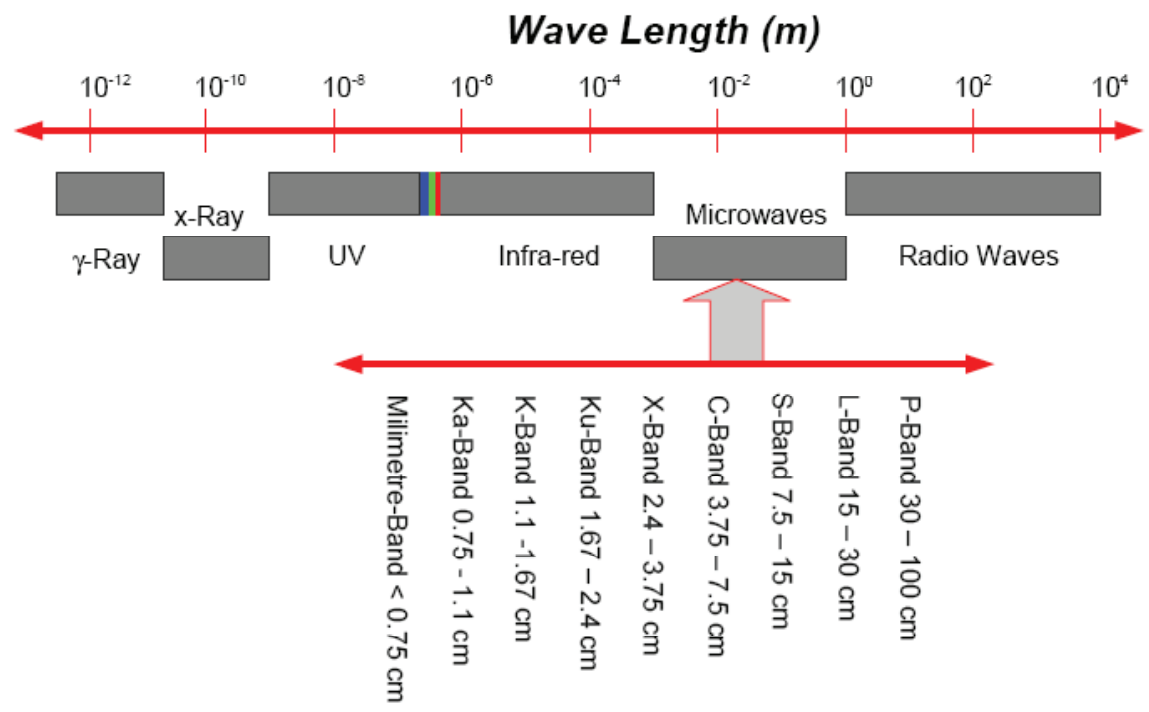

Figure 5.1: The electromagnetic spectrum, showing the microwave range in further detail.

devices (applicators) used to impose microwave fields onto dielectric materials and some applications of electromagnetic heating in biological systems.

\subsection{Microwave Frequency and its Influence over Microwave Heating}

Microwave frequencies occupy portions of the electromagnetic spectrum between $300 \mathrm{MHz}$ to $300 \mathrm{GHz}$. The full range of microwave frequencies is further subdivided into various bands, as indicated in Figure 5.1.

Because microwaves are also used in the communication, navigation and defence industries, their use in thermal heating is restricted to a small subset of the available frequency bands. Commonly used frequencies include $434 \pm 1 \mathrm{MHz}, 922 \pm 4 \mathrm{MHz}$, $2450 \pm 50 \mathrm{MHz}$ and $5800 \pm 75 \mathrm{MHz}$ (Commonwealth Department of Transport and Communications, 1991; International Telecommunication Union, 2004). In Australia, these frequencies have been set aside for Industrial, Scientific and Medical (ISM) applications (Commonwealth Department of Transport and Communications, 1991; International Telecommunication Union, 2004). All these frequencies interact to some degree with moist materials. 
Microwave heating depends on the ability of the microwave's electric field to polarise dipolar molecules (Metaxas \& Meredith, 1983). A dipole is essentially two equal and opposite charges separated by a finite distance. An example of this is the covalent bonds in a water molecule, giving the water molecule a dipole movement. Water is the typical case of a non-symmetric molecule, because there is a tendency for the larger oxygen atom to hold the two valance electrons more often than the two hydrogen atoms; therefore, inducing a slight negative change to the oxygen atom and a slight positive charge to the two hydrogen atoms. When this is coupled with the angular structure of the water molecule, there is a net positive end to the molecule and a net negative end. This results in a classic dipolar structure. Dipoles may be a natural feature of the dielectric or they may be induced (Kelly \& Rowson, 1995). Distortion of the electron cloud around non-polar molecules or atoms through the presence of an external electric field can also induce a temporary dipole movement.

The interaction with an oscillating external electric field associated with the microwave energy induces torque on polar molecules and the resulting movement generates friction inside the dielectric, which is dissipated as heat. Depending on the frequency, the dipole may move in time with the electric field, lag behind it, or remain apparently unaffected (Chaplin, 2004). When the molecular dipole movement lags the applied field, interactions between the dipole and the field lead to energy dissipation within the material and heating. The extent of heating depends on the phase difference between the applied fields and the dipole moment of the molecules. The ease with which dipole movement occurs depends on the viscosity and the mobility of the electron clouds within the molecule (Chaplin, 2004). In the case of water these, in turn, depend on the strength and extent of the hydrogen bonded networks within the liquid phase (Chaplin, 2004). In free liquid water this movement occurs at $\mathrm{GHz}$ frequencies whereas in more restricted 'bound' water it occurs at $\mathrm{MHz}$ frequencies and in ice it occurs at $\mathrm{kHz}$ frequencies (Chaplin, 2004).

\subsection{Electric Field Interactions with the Material and Their Influence over Microwave Heating}

Coupled with these polarisation effects, a dielectric can also exhibit direct conduction within the material, as charges are displaced by the applied fields (Metaxas \& Meredith, 1983). The combination of polarisation and conduction gives rise to displacement currents within the material (Torgovnikov, 1993). The complex displacement current can be resolved into a reactive component and a real component. To determine the current density in a dielectric material using Maxwell's equations and the physics of dielectric materials require the introduction of a complex dielectric constant $\varepsilon^{\star}$ (Debye, 1929). 
Debye (1929) deduced the well-known equation for the complex dielectric constant as:

$$
\varepsilon^{*}=\varepsilon^{\prime}+j \varepsilon^{\prime \prime}=\varepsilon_{\infty}+\frac{\varepsilon_{s}-\varepsilon_{\infty}}{1+j \omega \tau_{r}}
$$

The relative dielectric constant $\varepsilon$ ' expresses the material's ability to store electrical energy (Singh and Heldman, 1993 pp. 208) and thus represents the reactive nature of the material's electrical properties (Giancoli, 1989; Smith, 1976). In particular, $\varepsilon$ ' influences the wave impudence of the space occupied by the dielectric causing reflections at the inter-facial boundary between the air and the dielectric material. Changes in wave impedance also cause refraction of the wave due to the change in the propagation velocity of the microwave within the dielectric material compared with its velocity in air or vacuum (Montoro, Manrique, \& Gonzalez-Reviriego, 1999).

The dielectric loss $\varepsilon$ " represents the resistive nature of the material's electrical properties (Giancoli, 1989; Smith, 1976). Resistive losses within the medium reduce the amplitude of the microwave field and generate heat inside the material.

The dielectric properties of most materials are temperature, frequency and moisture dependent. For example, Torgovnikov (1993) states that macromolecules such as cellulose, hemi-cellulose and lignin, which make up the wood cell wall, are also subject to dipole polarisation. This is associated with the displacement of polar groups such as $\mathrm{OH}$ and $\mathrm{CH}_{2} \mathrm{OH}$ relative to the motionless parts of these macromolecules. In spite of this, dry wood, which is basically a mixture of these macromolecules, air, and bound water, does not interact very strongly with microwaves (Chaplin, 2004; Torgovnikov, 1993). On the other hand, free liquid water in wood structures such as tracheids and vessels interacts strongly with microwaves (Chaplin, 2004) and has been described as the "key substance attenuating microwaves" during microwave heating in many natural materials (Zielonka \& Gierlik, 1999).

Figure 5.2 shows the frequency and temperature dependency of the dielectric properties of free liquid water. It is interesting to note that the maximum dielectric loss occurs at much higher frequencies than those which are normally reserved for industrial microwave applications; however, the loss factor significantly increases with increasing concentrations of dissolved solids in the water, particularly salts. This is shown in Figure 5.3.

Because water plays such an important role in many organic systems, the dielectric properties of these materials is dependent on the water content of the samples (Figure 5.4).

In the particular case of anisotropic materials, such as wood, the orientation of the electric field vector has a significant effect on the dielectric properties of the material (Torgovnikov, 1993, pp. 13-17). Wood grain has a cylindrical geometry; therefore, within the wood structure there are three primary coordinates, corresponding to the normal cylindrical coordinate system. These are the radial direction running from the 


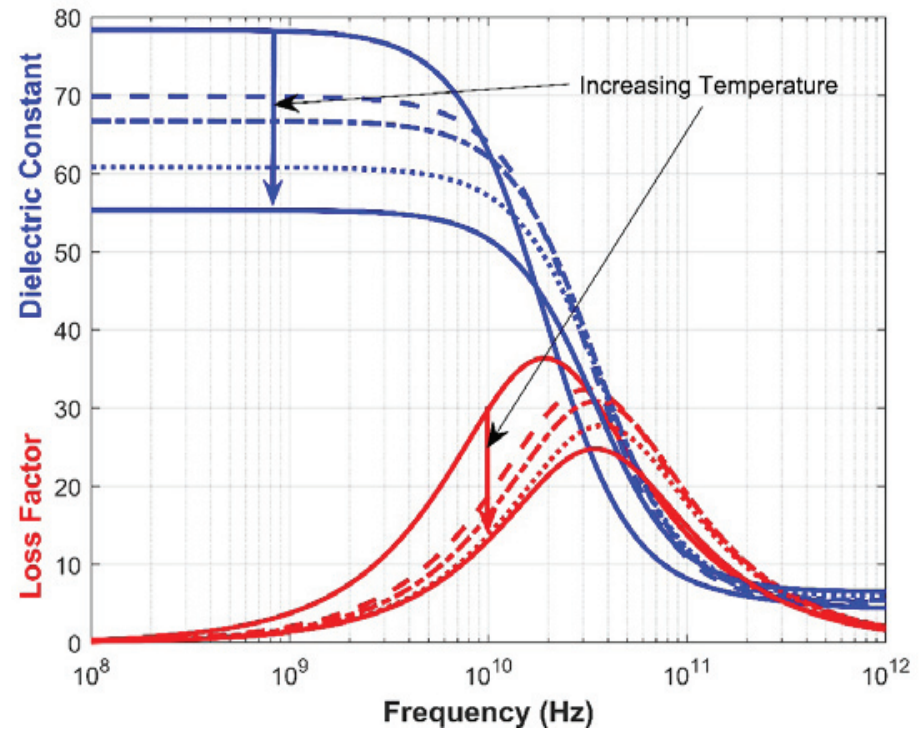

Figure 5.2: Dielectric constant and dielectric loss of water between $0^{\circ} \mathrm{C}$ and $100^{\circ} \mathrm{C}$, the arrows showing the effect of increasing temperature.

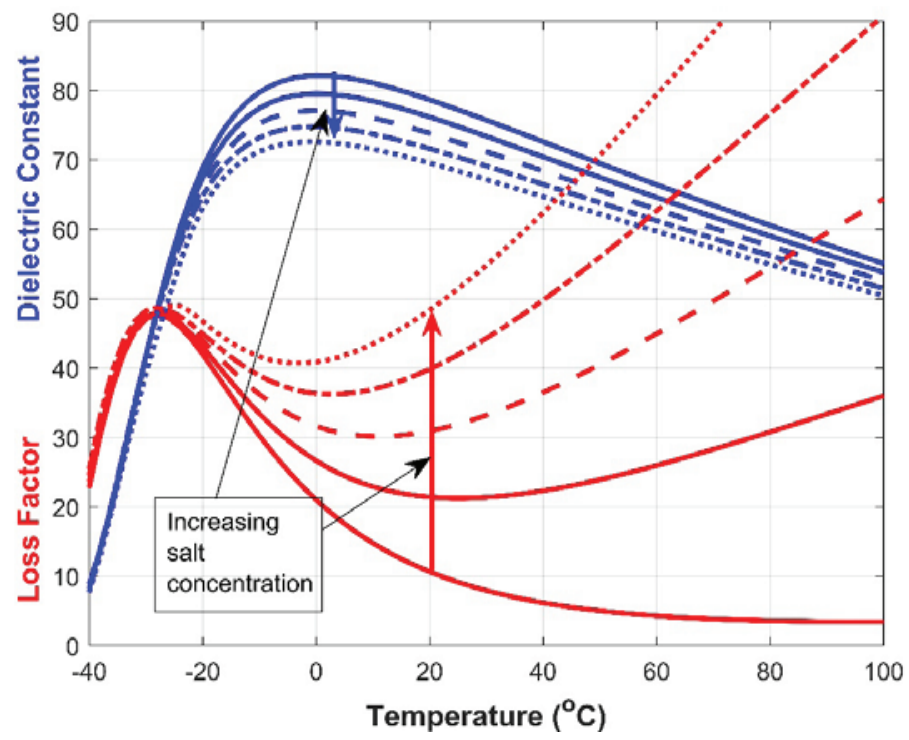

Figure 5.3: Dielectric constant and Loss Factor at $2.45 \mathrm{GHz}$ for different parts per thousand w/w (ppt) salinity for the range for $-20^{\circ} \mathrm{C} \sim+40^{\circ} \mathrm{C}$. 


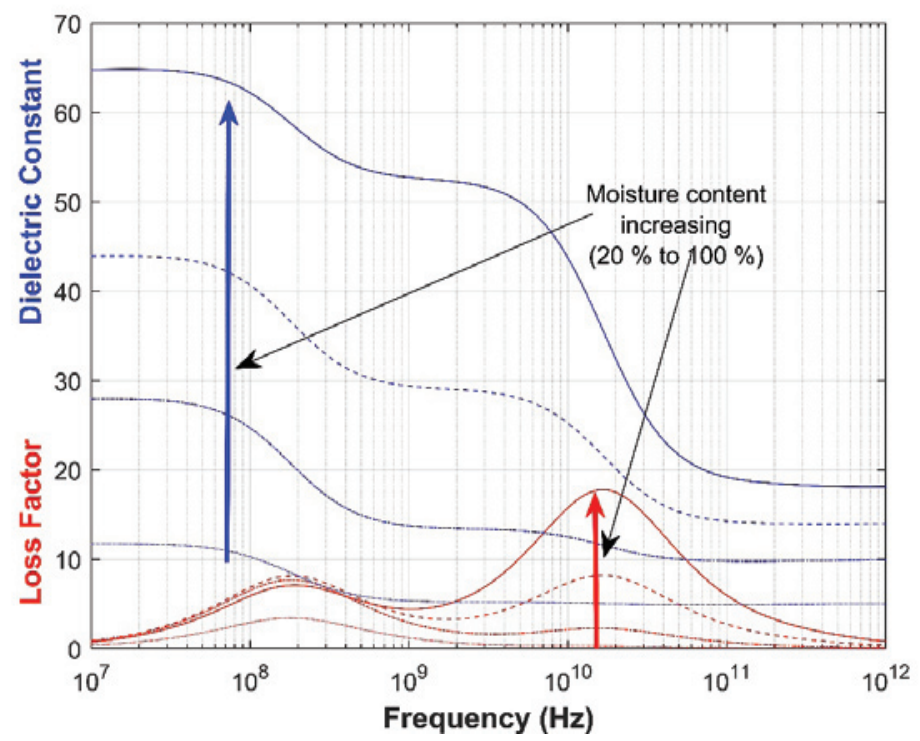

Figure 5.4: Dielectric properties of vegetative materials as a function of frequency and moisture content.

pith to the cambium, the tangential direction which is tangent to the growth rings and the longitudinal direction running along the length of the grain.

The power transmitted by an electromagnetic wave is proportional to the square of the electric field's magnitude (Giancoli, 1989). The electric field strength within a dielectric medium depends on the electrical field strength at the inter-facial surface between the material and the air, the reflection coefficient of this inter-facial surface, the geometry of the microwave applicator, the geometry of the material itself and the internal attenuation of the electric field with distance from the surface.

\subsection{Challenges Associated with Microwave Research}

According to McNamee and Chauhan (2009), there are several challenges when attempting to conduct high-quality RF and microwave research. Of primary importance is the interaction between the radiation and matter. If the effects of electromagnetic fields are of interest, the rate at which heat energy is applied to the sample must not exceed the rate at which it is removed; otherwise the temperature within the sample will rise and thermal confounding of the study may occur. If electromagnetic energy is applied to biological samples at a low rate (e.g. $<1 \mathrm{~W} \mathrm{~kg}^{-1}$ ) or for a short duration, then passive cooling may permit the tissue/body/sample temperature to remain within a 

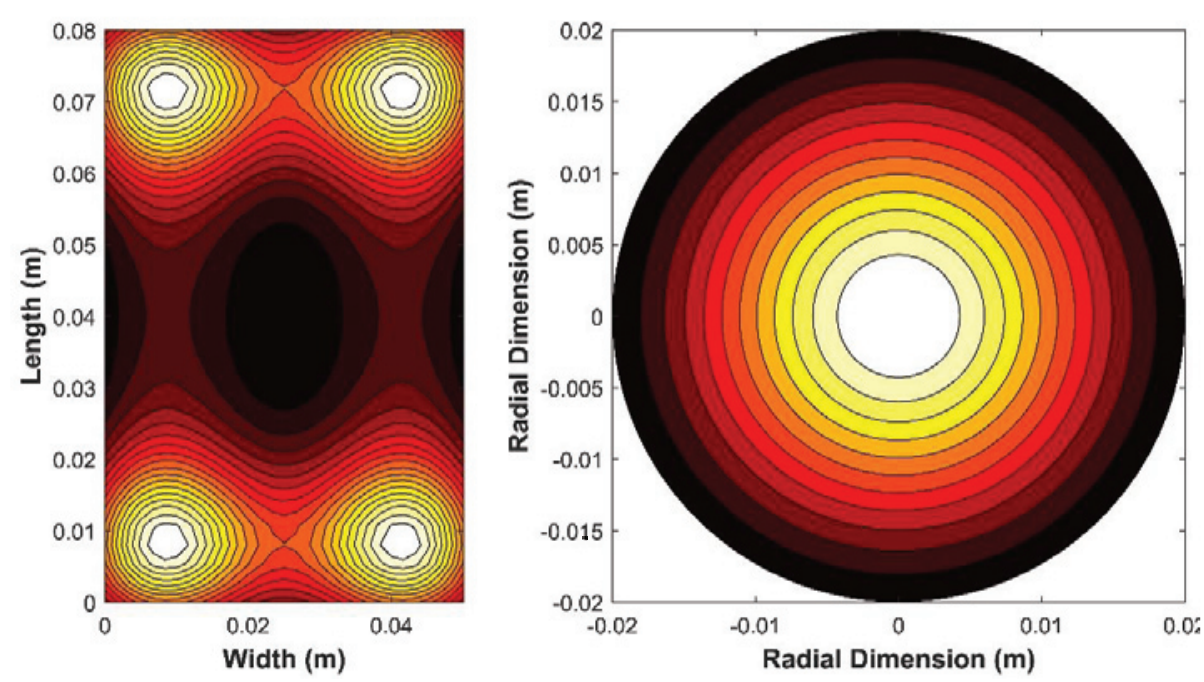

Figure 5.5: Temperature distribution in (left) cross section of a rectangular block and (right) cross section of a sphere with dielectric properties of $\mathrm{e}^{\prime}=9.6$ and e" $=2.5$ after 120 seconds of microwave heating at $2.45 \mathrm{GHz}$, based on models derived by Brodie (2008).

normal physiological range (McNamee \& Chauhan, 2009). However, if higher specific absorption rates $\left(>1 \mathrm{~W} \mathrm{~kg}^{-1}\right)$ are employed, then some form of active cooling mechanism may be required to ensure that excessive sample heating does not occur.

Another significant challenge is the non-homogeneity of energy absorption within the sample (McNamee \& Chauhan, 2009). The development of hot-spots due to the nature of microwave heating has been an ongoing concern for many researchers (Metaxas \& Meredith, 1983). The temperature distribution in a material undergoing RF or microwave heating is dependent on several factors; however, the geometry of the heated material itself tends to focus the electromagnetic energy into certain locations within the body of the heated object (Brodie, 2008; McNamee \& Chauhan, 2009). Figure 5.5 illustrates the focusing effect of microwave heating in rectangular blocks and spheres.

A related problem is the manifestation of thermal runaway in these hot-spots. Thermal runaway, which manifests itself as a sudden temperature rise due to small increases in the applied microwave power, is very widely documented (Nelson, Wake, Chen, \& Balakrishnan, 2001; Vriezinga, 1998; Zielonka \& Dolowy, 1998). It has also been reported after some time of steady heating at fixed power levels and is usually attributed to temperature dependent dielectric and thermal properties of the material.

In addressing the phenomenon of thermal runaway, Vriezinga (1998) used analytical solutions to the differential equations that describe heat diffusion 


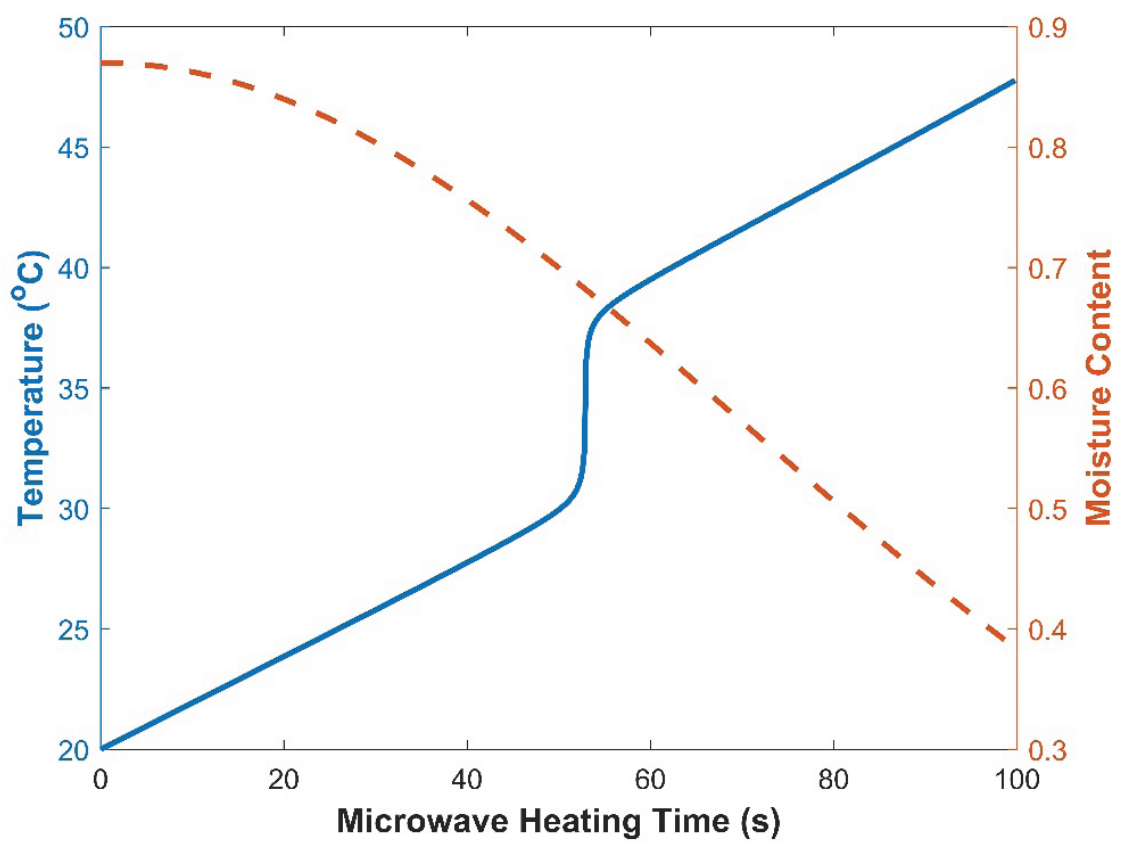

Figure 5.6: Example of thermal runaway in plant material dur to changing dielectric properties as the sample dries out.

(independent of moisture movement) in isothermal media to obtain S-shaped temperature versus microwave power curves. Hill and Marchant (1996) also developed S-shaped temperature versus microwave power curves during their investigation of microwave heating. They describe these curves as a multi-valued function of microwave power in which the upper and lower arms are stable, but the central arm is unstable. As the power increases from zero, the temperature stays on the lower arm until a critical power level is reached; then an infinitesimal increase in power will cause the temperature to jump to the upper arm. If the power is decreased the temperature will remain on the upper arm until a second critical power value is reached; then the temperature abruptly falls to the lower arm (Hill \& Marchant, 1996). Liu et al. (2003) reiterate this interpretation of these curves attributing the sudden jump in temperature to thermal runaway.

Figure 5.6 shows the temperature and moisture content in the centre of a plant stem of $10 \mathrm{~mm}$ diameter heated with microwave energy. In this case, the application of microwave heating dries the sample, which ultimately reduces the dielectric properties of the sample. As the dielectric properties reach a threshold value, the field can resonate inside the material and cause a sudden jump in temperature. Vriezinga 
(1998) also suggested that thermal runaway is a result of field resonance in the heated object.

\subsection{Non-Thermal Effects}

Various authors have proposed that changes in thermodynamic parameters under microwave irradiation, compared with those predicted by conventional heating, are caused by the "microwave effect" (De la Hoz, Díaz-Ortiz, \& Moreno, 2007). Microbiological studies involving microwave irradiation have resulted in the following two conflicting conclusions; cell death was solely the result of heat produced by microwave irradiation; death was due to not only heat but also microwave electric field intensity (Banik, Bandyopadhyay, \& Ganguly, 2003). The existence or otherwise of a "microwave effect" or non-thermal effect of microwave treatment is controversial.

Microwave photons have energies of the order of $10^{-5} \mathrm{eV}$ (Vollmer, 2004). Simple estimates easily show that the number of microwave photons within a commercial oven is orders of magnitude too small to establish multiphoton dissociation or ionization of the processed materials (Vollmer, 2004); therefore the probability that microwave processing is having a non-thermal effect on the thermodynamics of the system is small.

One possible explanation for the observed changes in thermodynamic parameters, which have been observed in experimental work, is heat and vapour coupling (Brodie, 2007). Very rapid heating and drying during microwave processing of moist materials have been widely reported (Ni, Datta, \& Parmeswar, 1999; Torgovnikov \& Vinden, 2009; Vinden \& Torgovnikov, 2000; Zielonka \& Dolowy, 1998). The movement of hot moisture through the material, under the influence of microwave heating, effectively increases the thermal diffusivity and drying rate of the system. It is reasonable to expect that the thermal diffusivity and drying rates are linked to the applied microwave power.

In experiments using wool fibres, described by Cassie, King and Baxter (1940 in Crank, 1979), the isothermal moisture diffusion coefficient predicted that moisture equilibrium should be reached within seconds of a sudden change in external humidity; however, their experiments demonstrated that equilibrium was only reached after an hour or more of exposure to the new external conditions (Crank, 1979). Henry (1948) explored this phenomenon and deduced that there was strong coupling between the thermal behaviour and moisture movement in these porous textiles. His work revealed that the combined processes of heat and vapour diffusion are equivalent to the independent diffusion of two quantities, each of which is a linear function of vapour concentration and temperature. The diffusion coefficients of these two quantities are always such that one is much less and the other much greater than would be observed, were vapour and heat diffusion not coupled together; therefore, 


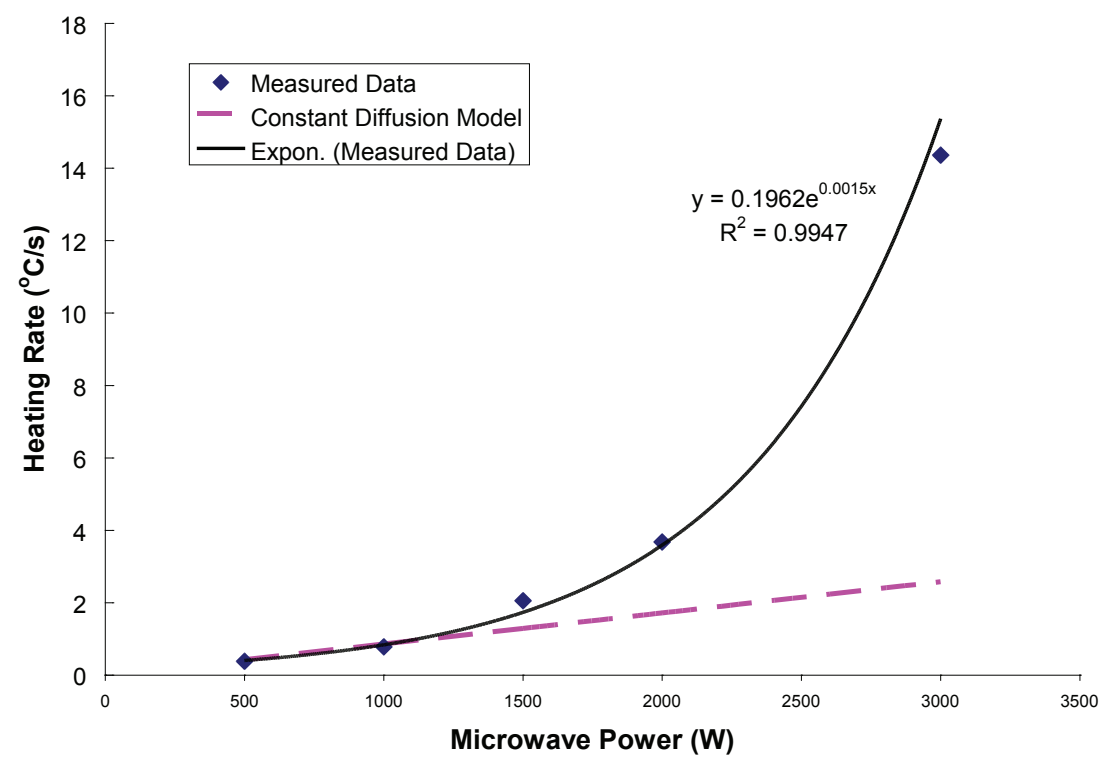

Figure 5.7: Heating rate in $25 \mathrm{~mm}$ by $25 \mathrm{~mm}$ Eucalyptus regnans samples, heated in a wave-guide, as a function of applied power (Dotted line represents the response that constant diffusion should produce).

the independent solution of the heat and vapour diffusion equations is inadequate to describe their combined influence (Henry, 1948).

The diffusion coefficient for the slower quantity of the coupled system is always less than either the isothermal moisture diffusion constant or the constant vapour concentration coefficient for heat diffusion, whichever is less, but never by more than one half (Henry, 1948). The faster diffusion coefficient may be many times greater than either of the independent diffusion constants (Henry, 1948).

Henry (1948) presents a nomogram relating the fast diffusion coefficient to the default diffusion coefficient for the material at $20^{\circ} \mathrm{C}$ and $65 \%$ relative humidity. This nomogram can be used to forecast the thermal diffusivity of the system under different conditions. For example, if the relative humidity remains constant and the temperature of some part of the system rapidly rises to $55^{\circ} \mathrm{C}$, Henry's nomogram suggests that the diffusion coefficient for the faster wave will be about 7.5 times higher than the standard thermal diffusivity of the material. Figure 5.7 shows the relationship between heating rates and applied microwave power during microwave heating experiments involving Eucalyptus regnans wood samples, which were conducted at the University of Melbourne. It would be tempting to attribute the non-linear response of the samples to a "microwave effect"; however, it may be better to regard this as 
a progressively stronger coupling between the thermal and vapour diffusion in the samples, as the applied microwave power increases.

\subsection{References}

Adamski, W., \& Kitlinski, M. (2001). On measurements applied in scientific researches of microwave heating processes. Measurement Science Review, 1(1), 199-203.

Banik, S., Bandyopadhyay, S., \& Ganguly, S. (2003). Bioeffects of microwave--a brief review. Bioresource Technology, 87(2), 155-159.

Bond, E. J., Li, X., Hagness, S. C., \& Van Veen, B. D. (2003). Microwave imaging via space-time beamforming for early detection of breast cancer. IEEE Transaction on Antennas and Propagation, 51(8), 1690-1705.

Brodie, G. (2007). Simultaneous heat and moisture diffusion during microwave heating of moist wood. Applied Engineering in Agriculture, 23(2), 179-187.

Brodie, G. (2008). The influence of load geometry on temperature distribution during microwave heating. Transactions of the American Society of Agricultural and Biological Engineers, 51(4), 1401-1413.

Chaplin, M. (2004). Water Structure and Behavior. Retrieved from http://www.lsbu.ac.uk/water/ index.html

Commonwealth Department of Transport and Communications. (1991). Australian Radio Frequency Spectrum Allocations. Commonwealth Department of Transport and Communications.

Crank, J. (1979). The Mathematics of Diffusion. Bristol: J. W. Arrowsmith Ltd.

De la Hoz, A., Díaz-Ortiz, A., \& Moreno, A. (2007). REVIEW ON NON-THERMAL EFFECTS OF MICROWAVE IRRADIATION IN ORGANIC SYNTHESIS. Journal of Microwave Power \& Electromagnetic Energy, 41(1), 1.44.

Debye, P. (1929). Polar Molecules. New York: Chemical Catalog.

Gallawa, J. C. (1998). The Complete Microwave Oven Service Handbook: Operation, Maintenance, Troubleshooting and Repair. Englewood Cliffs: Prentice Hall.

Giancoli, D. C. (1989). Physics for Scientists and Engineers (2nd ed.). New Jersey: Prentice Hall.

Henry, P. S. H. (1948). The diffusion of moisture and heat through textiles. Discussions of the Faraday Society, 3, 243-257.

Hill, J. M., \& Marchant, T. R. (1996). Modelling Microwave Heating. Applied Mathematical Modeling, 20(1), 3-15.

Holman, J. P. (1997). Heat Transfer (8th ed.). New York: McGraw-Hill.

International Telecommunication Union. (2004). Spectrum Management for a Converging World: Case Study on Australia. Retrieved from

Kelly, R. M., \& Rowson, N. A. (1995). Microwave reduction of oxidised ilmenite concentrates. Minerals Engineering, 8(11), 1427-1438.

Liu, B., Marchant, T. R., Turner, I. W., \& Vegh, V. (2003). A comparison of semi-analytical and numerical solutions for the microwave heating of lossy material in a three-dimensional waveguide. Paper presented at the Third World Congress on Microwave and Radio Frequency Applications, Sydney.

McNamee, J. P., \& Chauhan, V. (2009). Radiofrequency radiation and gene/protein expression: A review. Radiation Research, 172(3), 265-287.

Metaxas, A. C., \& Meredith, R. J. (1983). Industrial Microwave Heating. London: Peter Peregrinus.

Montoro, T., Manrique, E., \& Gonzalez-Reviriego, A. (1999). Measurement of the refracting index of wood for microwave radiation. Holz als Roh- und Werkstoff, 57(4), 295 -299. 
Nelson, M. I., Wake, G. C., Chen, X. D., \& Balakrishnan, E. (2001). The multiplicity of steady-state solutions arising from microwave heating. I. Infinite Biot number and small penetration depth. The ANZIAM Journal, 43(1), 87-103.

$\mathrm{Ni}$, H., Datta, A. K., \& Parmeswar, R. (1999). Moisture loss as related to heating uniformity in microwave processing of solid foods. Journal of Food Process Engineering, 22(5), 367-382.

Perre, P., \& Turner, I. W. (1999). The use of numerical simulation as a cognitive tool for studying the microwave drying of softwood in an over-sized wave-guide. Wood Science and Technology, 33(6), 445-464.

Smith, R. J. (1976). Circuits, Divices and Systems (3rd ed.). New York: Wiley International.

Torgovnikov, G., \& Vinden, P. (2009). High-intensity microwave wood modification for increasing permeability. Forest Products Journal, 59(4), 84-92.

Torgovnikov, G. I. (1993). Dielectric Properties of Wood and Wood-Based Materials. Berlin: SpringerVerlag.

Van Remmen, H. H. J., Ponne, C. T., Nijhuis, H. H., Bartels, P. V., \& Herkhof, P. J. A. M. (1996). Microwave Heating Distribution in Slabs, Spheres and Cylinders with Relation to Food Processing. Journal of Food Science, 61(6), 1105-1113.

Vinden, P., \& Torgovnikov, G. (2000). The physical manipulation of wood properties using microwave. Paper presented at the International Conference of IUFRO, Tasmania, Australia.

Vollmer, M. (2004). Physics of the microwave oven. Physics Education, 39(1), 74-81.

Vriezinga, C. A. (1998). Thermal runaway in microwave heated isothermal slabs, cylinders, and spheres. Journal of Applied Physics, 83(1), 438 -442.

Zhao, H., Turner, I. W., \& Torgovnikov, G. (1998). An experimental and numerical investigation of the microwave heating of wood. The Journal of Microwave Power and Electromagnetic Energy, 33(2), 121-133.

Zielonka, P., \& Dolowy, K. (1998). Microwave Drying of Spruce: Moisture Content, Temperature and Heat Energy Distribution. Forest Products Journal, 48(6), 77-80.

Zielonka, P., \& Gierlik, E. (1999). Temperature distribution during conventional and microwave wood heating. Holz als Roh- und Werkstoff, 57(4), 247-249. 